\title{
Work in Progress: A Qualitative Exploration of Female Undergraduate Decisions to Specialize within Engineering Disciplines
}

\author{
Dr. M. Teresa Cardador, University of Illinois at Urbana-Champaign \\ Prof. Karin Jensen, University of Illinois at Urbana - Champaign
}

Karin Jensen, Ph.D. is a Teaching Assistant Professor in bioengineering at the University of Illinois at Urbana-Champaign. Her research interests include student mental health and wellness, engineering student career pathways, and engagement of engineering faculty in engineering education research. She was awarded a CAREER award from the National Science Foundation for her research on undergraduate mental health in engineering programs. Before joining UIUC she completed a post-doctoral fellowship at Sanofi Oncology in Cambridge, MA. She earned a bachelor's degree in biological engineering from Cornell University and a Ph.D. in biomedical engineering from the University of Virginia.

\section{Dr. Kelly J Cross, University of Nevada, Reno}

Dr. Cross is currently an Assistant Professor in the Chemical and Materials Engineering Department at the University Nevada Reno. After completing her PhD in Engineering Education at Virginia Tech in 2015, Dr. Cross worked as a post-doctoral researcher with the Illinois Foundry for Innovation in Engineering Education and in the Department of Bioengineering with the Revolutionizing Engineering Departments (RED) grant at the University of Illinois at Urbana-Champaign. Dr. Cross' scholarship investigated student teams in engineering, faculty communities of practice, and the intersectionality of multiple identity dimensions. Her research interests include diversity and inclusion in STEM, intersectionality, teamwork and communication skills, assessment, and identity construction. Her teaching philosophy focuses on student centered approaches such as culturally relevant pedagogy. Dr. Cross' complimentary professional activities promote inclusive excellence through collaboration.

Ms. Grisel Lopez-Alvarez, University of Illinois at Urbana-Champaign 


\section{Work in Progress: A Qualitative Exploration of Female Undergraduate Decisions to Specialize within Engineering Disciplines}

\section{Introduction}

While engineering has long been recognized as one of the most highly and persistently sex segregated occupations in the US, researchers have also begun to recognize patterns of intraoccupational sex segregation within engineering, such that gendered roles and career paths exist in the engineering profession [1-3]. Men are more frequently in the most technical roles (i.e., those that rely almost exclusively on technical rather than professional skills). These roles are often perceived as the highest status and most characteristic of "real engineering" (and are also stereotypically masculine), and women in the less technical roles that are perceived as lower status and are stereotypically feminine $[1,4,5]$. This under-representation of women in the most technically-oriented roles within engineering may be problematic given that female engineers in more technically-oriented career paths have better retention and wage equality outcomes [2, 3, $6]$. Despite the benefits to female engineers of remaining in technical roles and career paths within the profession, there is evidence that women are more likely than men to be engaged in more "social" versus "technical" work activities [3], and to rate themselves lower than men on professional identity traits valued in engineering (e.g., problem solving and technical leadership) [7]. While researchers have noted patterns of intra-occupational sex segregation in engineering, to our knowledge minimal research has examined when and why these gendered career patterns begin to emerge, nor have researchers systematically examined the gendering of elective tracks in engineering education.

In this research, we explore one possible "upstream" antecedent--female engineering students' elective track selection during undergraduate education-because this is a decision that shapes the remainder of their engineering education and preparation, and should thus have important implications for their downstream career attitudes and decisions. Specifically, we employ a longitudinal, multi-method, and multi-level case study methodology to systematically examine whether, how and why elective track choices become gendered during undergraduate engineering education, and what the implications might be. To explore the possible influence of elective track selection on career attitudes and decisions, the present study seeks to identify to what extent female and male engineering undergraduates in one university setting pursue different elective tracks within engineering majors, to pinpoint the personal and structural factors contributing to female students track selection, and to link elective track decisions with career choices post-graduation. Toward these goals, we are collecting institutional data and conducting multi-year surveys and interviews with engineering department faculty, staff, advisors, and female students. By understanding how and why elective tracks become gendered within engineering majors, we hope to identify effective strategies for improving the preparation of women to pursue technical roles and career paths in their chosen field of study, with the broad goal of improving the workplace retention of female engineers.

As this project is ongoing, we plan to present preliminary results based on wave one (1) interviews with 30 female engineering students in their sophomore year (the year when elective track selections are made). 


\section{Project Background and Theoretical Framework}

Existing research shows that men and women in gender-typed occupations - such as engineering - often experience their professional roles differently [8]. In a recent study Cech (2015) found that as compared to men's professional identity, women's professional identities were less likely to emphasize dominant and valued traits in engineering such as technical leadership and concurrently, more likely to emphasize typically marginalized traits (e.g., social consciousness) [7]. Further, these gender differences in engineering identity traits were linked to gender differences in persistence in engineering education and wage gaps [3]. Others have noted the tendency among engineers to define "real" engineers in terms of technical "nuts and bolts" and scientific and mathematical expertise or rigor [9], thereby locating the less technical (i.e., social; [10]) aspects outside of what it means to be a "real" engineer [11, 12]. Godfrey (2014) found that there is a "devaluing of content or subject areas that were seen as 'easy' or 'soft' [13]. This extant research suggests that "real engineering" is often positioned as technical, and that women are less likely to adopt or perform the most celebrated (i.e., technical) engineering roles, with implications for greater risk of attrition $[1,2,7]$. Some previous scholars have considered the technical social dualism in engineering (e.g. [14], [15], [5]), however, our understanding of the role of major and within major track selection linked to career choices in engineering is understudied. To address this gap, the proposed research seeks to shed light on early career stage (i.e., "upstream") antecedents of gendered career patterns and their downstream career path/role choice. We use two complementary theoretical lenses to inform our investigation - intraoccupational sex segregation (IOSS), and social cognitive career theory (SCCT).

\section{Intra-Occupational Sex Segregation}

As noted in the introduction, while scholars are beginning to recognize forms of intraoccupational sex segregation (IOSS) in engineering, and to acknowledge that they may have important negative consequences for women's professional retention in engineering, there is a paucity of research examining how and why these gendered career patterns begin to emerge. Research on the medical profession provides some insights. This research points to an occupational prestige hierarchy as foundational to choice of specialty between and within medical subspecialties. Hinze (1999) shows that sub-specialties with the most prestige (e.g., surgery) are equated with stereotypical masculine characteristics - toughness, working long hours, and competitiveness - and occupied primarily by men. In contrast, sub-specialties with the lowest prestige (e.g., psychiatry) were associated with stereotypically feminine characteristics (e.g., empathy, effective communication) and occupied more by women [16]. This pattern is noted within sub-specialty as well. For example, researchers have found that even within the surgical subspecialty, women appear to gravitate toward what might be seen as the "softer" niches of the discipline (e.g., breast surgery vs. trauma surgery) [17].

Research suggests that this same occupational prestige hierarchy exists in engineering [1, 5, 8], whereby the most technical roles (e.g., technical specialists, technical fellows) are perceived as the highest status and most characteristic of "real engineering" (and also the most stereotypically masculine), and the less technical roles (e.g., business analysis, technical supervision, product management, etc.) are seen as "at the periphery" of engineering and as more stereotypically feminine [1]. This has been offered as a potential explanation for why women in engineering 
choose and/or are directed toward less technical roles and career paths [1]. What is not wellunderstood, however, is when female engineers' decisions about role and career path begin to formulate and whether/how factors present in their undergraduate educational environment may influence their later decisions to consider, or conversely gravitate away from, the most technical roles in their engineering major and sub-field. Accordingly, the purpose of this research is to examine how and why elective track choice - a factor significant to the formation, socialization, and preparation of engineers for the workforce - may become gendered during undergraduate education, and what the implications might be for downstream career decisions and attitudes.

\section{Social Cognitive Career Theory}

Social cognitive career theory (SCCT), an oft referenced literature in engineering education, has been used to explain the formation of career-relevant interests, choice, and attainment of careerrelated goals, and emphasizes the role of self-efficacy and outcome expectations (e.g., [18]; [19]; [20]; [21]). The theory posits that self-efficacy and outcome expectation beliefs are impacted by personal accomplishments, and contextual factors present in academic and career-development environments which impact personal behavior and choice [18]. In a "virtuous cycle" of sorts, self-efficacy (confidence) in one's abilities in the domain prompts positive initiation and expectations in career-related endeavors and interests are increased for those areas in which one feels efficacious [22].

This prior research suggests that SCCT is a valuable lens through which to examine how female engineering students make career-critical, elective track decisions during their undergraduate education, and the personal and structural (i.e., contextual) factors that may affect these decisions via positive technical outcome expectations. Further, SCCT informs an intersectional perspective with respect to gender and race [23, 24]. Accordingly, our study pays attention to the role of these factors of contributing to female engineers' elective track decisions and subsequent implications. Drawing from theories of IOSS and SCCT, we developed a conceptual model to inform our investigation (see Figure 1).

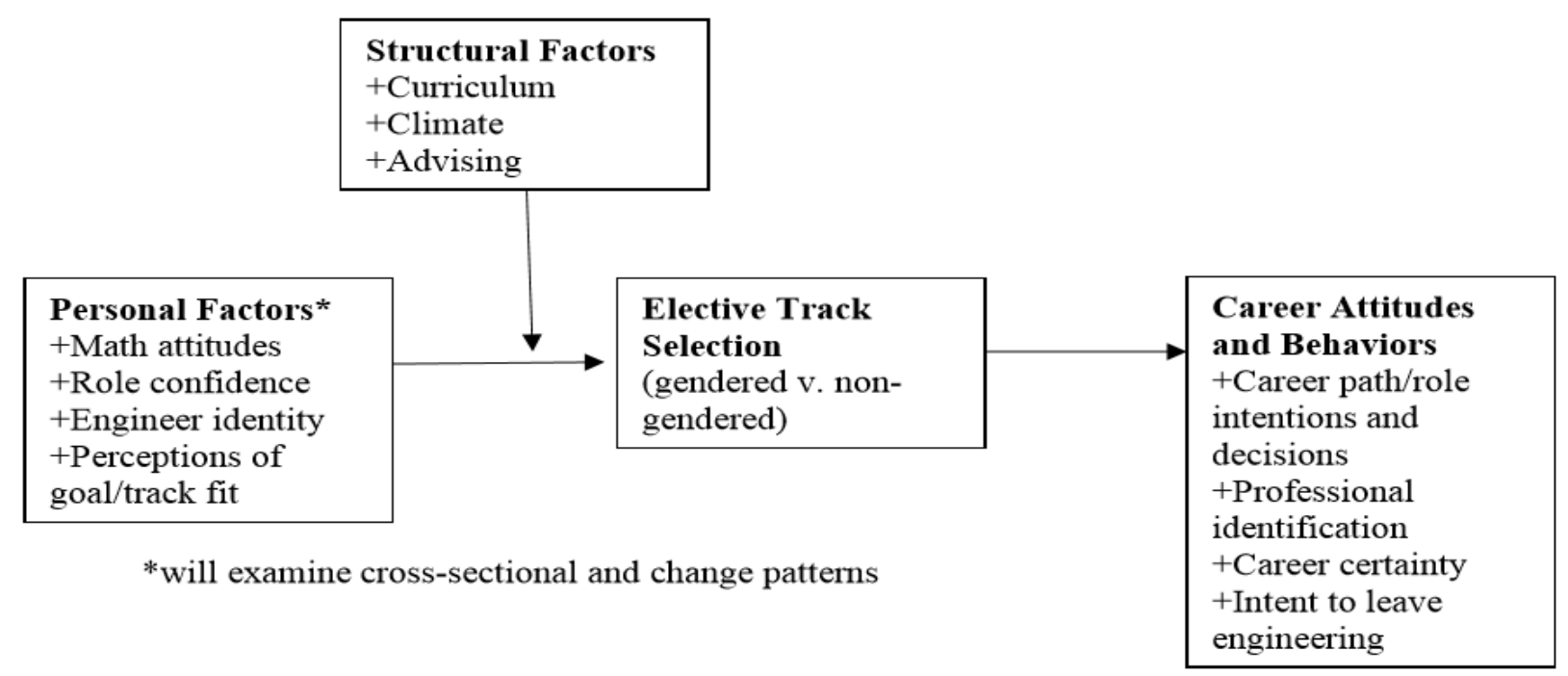

Figure 1. Conceptual Model for the Broader Study. 


\section{Methods}

We conducted interviews with 30 female engineers, 10 from each of three engineering majors Bioengineering, Computer Science, and Electrical Engineering. These three majors were selected based on the gender balance in each major - high (Bioengineering; 51.4\% female), medium (Computer science; $27.5 \%$ female) and low (Electrical Engineering; $14.2 \%$ female) at the focal institution.

\section{Qualitative Interview Design}

The interview protocol included three main sections. In the first section, students were asked about their major selection and influences on deciding on that major. For example, "In thinking about how you selected your major, who, if anyone, contributed to your choice? What courses, if any, prepared you for your major?" Participants were also asked to reflect on factors or characteristics that are required for success in the major, as well as the importance of technical and professional skills. In the second section of questions, participants were asked about their specialization or track in their major (if enrolled in a major where tracks are offered) or their opinion of why the major does not require tracks (if enrolled in a major where tracks are not offered). In both cases, participants were asked about how students can specialize in their discipline outside of coursework. In the final set of questions, participants were asked questions about their post-graduation plans. For example, "What are your plans for internships or career development in the near future?" Students were also asked about the career path they envisioned, particularly whether they saw themselves in a more technical or managerial role. Finally, at the conclusion of the interview, participants were offered the opportunity to share any additional information or thoughts that had not been covered in the interview.

\section{$\underline{\text { Data Collection }}$}

Data were collected in the fall 2019 semester under approval of the institution IRB. Participants were recruited by email from advisors of the Electrical Engineering, Bioengineering, and Computer Science majors. Participants were eligible if they identified as female, were in their second year of undergraduate education, and were currently enrolled in one of the three focal majors. Participants were informed in the recruitment email that they would receive $\$ 20$ for their participation. All interviews were held face-to-face in a private office on the engineering quad. Interviews, which lasted an average of 45 minutes, were conducted by one of three project team interviewers. The interviews were audio recorded and transcribed verbatim.

\section{Data Analysis}

Interview transcripts were cleaned to remove identifying information. We next analyzed the cleaned transcripts using a grounded theory thematic analysis approach. All qualitative analysis was performed in MaxQDA software. During the first cycle of coding, two of the researchers each coded 10 transcripts (distributed across the three majors) and performed open coding. The open codes were aggregated into categories which were then used to develop a codebook for use in coding the remaining interviews. 


\section{Results}

Though data analysis is ongoing, the preliminary findings from 20 of the wave 1 student interviews point to at least four factors most relevant to female engineers' elective track selection. First, data show a strong influence of peer (vs. advisor or instructor) influence on elective choices. Second, data show that students perceive the culture to be intense and competitive, and that peer comparison is a constant for most students. Third, themes from the data indicate that both interest and anticipated performance may be key factors in in selecting electives and elective tracks. Fourth, the majority of women sampled showed at least some interest in managerial/professional roles and careers in engineering at this stage of their careers. We provide a brief overview of each of these sets of findings.

First, with respect to peer influence, female engineers in all three majors report relying heavily on recommendations from friends in their major, more senior peers, and peer posts on social media (e.g., Reddit) to inform their decisions about which electives to take and which ones to avoid. Commonly cited reasons for avoiding courses were excessive workload and anticipated low grades. In contrast, academic and faculty advisors appeared to have very little influence in elective and elective track selection. One student described what many others echoed: "I don't really utilize my advisor that often. Usually just to set up my classes and stuff." (B104)

Second, the strong presence of peer comparison, particularly in the Computer Science and Electrical Engineering majors, was another theme to emerge from preliminary analysis. The majority in the Computer Science and Electrical Engineering majors described the peer culture as "intensive" and "competitive". Many described significant stress associated with their failure to "get all As" in their first and second years (something that most were used to in high school), and felt that they lacked at least some of the criteria for success in their major. Most participants were keenly aware of their performance compared to their peers and many felt that the most successful students "were working all the time" or that "good students have no outside interests". Some describe this this intensive culture associated with the first two years of course work as related to their desire to gravitate toward electives in subfields where they felt they were a "more natural fit". Students appeared less interested in topics/subfields which they felt they were "not naturally good at" or where they felt they "lagged behind" their peers in capability. Despite a culture of learning and mastery, students we interviewed made repeated reference to a somewhat fixed learning orientation, particularly when it came to technical knowledge and skills. Technical knowledge and skills were considered more "innate" - i.e., either you "get it" or you don't—as compared to professional skills, which students felt could be more easily learned. Somewhat paradoxically, many also noted that whether you understood something well or not was also based on how much prior exposure you may have had/not had to the topic.

Third, interest and anticipated performance also seemed related to elective track selection. Students based their elective selection on personal interest (how interesting they found the topic), and how much they enjoyed previous courses in a particular specialization area (e.g., hardware vs. software or biomechanics vs. therapeutics). Related to the second theme, however, interest also appeared related to how well one performed in a particular course. 
A fourth theme to emerge from the wave 1 student data was that approximately fifty percent of informants had some plan to pursue managerial roles, rather than purely technical roles postgraduation. Some explained wanting to "take the managerial path", "go into project management", "get a business minor", or "get an MBA". Reasons given for these plans were fit with interest in "roles that require professional skills", wanting a change from competitive and "intense technical work", and viewing these types of roles as more highly regarded in the profession.

Table 1. Illustrative Quotes for Emergent Themes.

\begin{tabular}{|c|c|c|}
\hline Theme & $\begin{array}{l}\text { Theory } \\
\text { (IOSS/SCCT) }\end{array}$ & Quote \\
\hline $\begin{array}{l}\text { Peer } \\
\text { Influence }\end{array}$ & SCCT & $\begin{array}{l}\text { I talked to my advisor before but I didn't talk too much with } \\
\text { my advisor. I think some people really talk to advisor to get } \\
\text { the advice, but for me it's, I talk to my friends. So those who } \\
\text { have, they have taken the four level courses. So I talked to my } \\
\text { friends who have taken the classes I'm interested in. So I will } \\
\text { say like, "What we will learn in this path?" And then I will get } \\
\text { the idea, "Okay, what I need to learn? And what's the most } \\
\text { important skill I need to have for this path?" (EE106) }\end{array}$ \\
\hline $\begin{array}{l}\text { Peer } \\
\text { Competition }\end{array}$ & SCCT/IOSS & $\begin{array}{l}\text { If we have a test or something you'll hear people say, 'Oh that } \\
\text { was so easy,' even though to me and might not be the most } \\
\text { easy test. (B105) } \\
\text { So in those competitive environments, I think everyone else } \\
\text { affects you. When they moving forward, you have to move } \\
\text { forward too. (EE105) }\end{array}$ \\
\hline $\begin{array}{l}\text { Personal } \\
\text { Interest }\end{array}$ & SCCT/IOSS & $\begin{array}{l}\text { I know on our bioengineering website, we have all the tracks } \\
\text { listed out with their description. So, that helps you gauge } \\
\text { where you want to fall. But, specifically with me, I feel like it } \\
\text { was more interest motivated than other things. And also, with } \\
\text { wanting to going into industry, I felt I would have more room } \\
\text { to grow if I was doing biomechanics in industry. (B104) }\end{array}$ \\
\hline $\begin{array}{l}\text { Managerial } \\
\text { Roles }\end{array}$ & IOSS & $\begin{array}{l}\text { The areas that I want to go into with computer science would } \\
\text { definitely enter that can require more professional skills } \\
\text { because being able to explain what you've done to non- } \\
\text { technical people and things that is very important. And some } \\
\text { things that are very important and interviews and talking to } \\
\text { managers and stuff. Actually being able to explain what } \\
\text { you've done is pretty important, regardless. But some fields } \\
\text { are definitely more of a ... you must be able to talk about it a } \\
\text { lot versus you're just working backend and the only person } \\
\text { you have to explain it to is your manager who is also a } \\
\text { technical. (CS106) }\end{array}$ \\
\hline
\end{tabular}




\section{Discussion and Future Work}

Based on preliminary results from interviews with 20 undergraduate female engineers in three separate majors, we noted several factors appear to have relevance for elective choice and elective track selection. Peer feedback and comparison appears integral to how students experience the major and appears related to how they make decisions about which electives to take and make assessments about what they are "naturally good at". Students rely minimally, if at all, on feedback from advisors or instructors in making elective selections. Although students do make selections based on interests, it is worth noting that assessments of anticipated performance can influence perceptions of interest. Finally, a majority of the students interviewed report wanting to pursue a role or career path that emphasizes professional/managerial skill in addition to technical skills. This is despite many reports that professional skills are not heavily emphasized in their major.

Our next steps are as follows. First, using the codebook developed by the first and second authors based on open coding of the first 20 interviews, two research assistants will re-code the same 20 interviews, and the first and second study authors will code the remaining 10 interviews. Inter-rater reliability will be established. Simultaneously, the authors and research assistants will begin analyzing interviews from 9 faculty and staff across the three majors, following the same process of analysis. Second, based on analyses of interview data from wave 1, we will develop a theoretical model that describes personal and structural influences on elective track selection and possible career implications. Third, this model will form the basis for development of a survey questionnaire that will be administered to a larger sample of engineering studies in fall 2020 in order to quantitatively test the relationships observed in the qualitative data.

\section{Acknowledgements}

This material is based upon work supported by the National Science Foundation under Grant No. 1848498. Any opinions, findings, and conclusions or recommendations expressed in this material are those of the authors and do not necessarily reflect the views of the National Science Foundation. The authors also wish to thank Dr. Elizabeth Litzler, the Project Evaluator, for her valuable input. Additionally, we thank the students, advisors and faculty who participated in the study for sharing their experiences.

\section{References}

[1] M. T. Cardador, "Promoted Up But Also Out? The Unintended Consequences of Increasing Women's Representation in Managerial Roles in Engineering," Organization Science, vol. 28, pp. 597-617, 2017.

[2] M. T. Cardador and P. L. Hill, "Career paths in engineering firms: Gendered patterns and implications," Journal of Career Assessment, p. 1069072716679987, 2016.

[3] E. A. Cech, "Ideological wage inequalities? The technical/social dualism and the gender wage gap in engineering," Social Forces, vol. 91, pp. 1147-1182, 2013.

[4] L. Bailyn, "Experiencing technical work: A comparison of male and female engineers," Human Relations, vol. 40, pp. 299-312, 1987. 
[5] W. Faulkner, "Dualisms, hierarchies and gender in engineering," Social studies of science, vol. 30, pp. 759-792, 2000.

[6] K. L. Milkman, M. Akinola, and D. Chugh, "What happens before? A field experiment exploring how pay and representation differentially shape bias on the pathway into organizations," Journal of Applied Psychology, vol. 100, p. 1678, 2015.

[7] E. Cech, "Engineers and engineeresses? Self-conceptions and the development of gendered professional identities," Sociological Perspectives, vol. 58, pp. 56-77, 2015.

[8] J. K. Fletcher, "Disappearing acts," Gender, Power, and Relational Practice at Work. Massachusetts Institute of Technology, Cambridge, 1999.

[9] D. Riley, "Rigor/Us: Building boundaries and disciplining diversity with standards of merit," Engineering Studies, vol. 9, pp. 249-265, 2017.

[10] W. Faulkner, "Gender in and of Technology," in Science, Technology and Society International Symposium, Istanbul, Istanbul Technical University, Institute of Social Sciences Publications, 1999.

[11] J. Trevelyan, "Mind the gaps: engineering education and practice," in Proceedings of the 21 st Annual Conference for the Australasian Association for Engineering Education, 2010, p. 383.

[12] K. L. Tonso, On the outskirts of engineering: Learning identity, gender, and power via engineering practice: Brill Sense, 2007.

[13] E. Godfrey, "Understanding disciplinary cultures: The first step to cultural change," Cambridge Handbook of Engineering Education Research, pp. 437-455, 2014.

[14] E. A. Cech and T. J. Waidzunas, "Navigating the heteronormativity of engineering: The experiences of lesbian, gay, and bisexual students," Engineering Studies, vol. 3, pp. 1-24, 2011.

[15] L. Leyva, J. Massa, and D. Battey, "Queering engineering: A critical analysis of the gendered technical/social dualism in engineering and engineering education research," in ASEE Annual Conference \& Exposition, 2016.

[16] S. W. Hinze, "Gender and the body of medicine or at least some body parts:(Re) constructing the prestige hierarchy of medical specialties," The Sociological Quarterly, vol. 40, pp. 217-239, 1999.

[17] G. Davis and R. Allison, "Increasing representation, maintaining hierarchy: An assessment of gender and medical specialization," SOCIAL THOUGHT \& RESEARCH: A Continuation of the Mid-American Review of Sociology, pp. 17-45, 2013.

[18] R. W. Lent, S. D. Brown, and G. Hackett, "Contextual supports and barriers to career choice: A social cognitive analysis," Journal of counseling psychology, vol. 47, p. 36, 2000.

[19] R. W. Lent, H.-B. Sheu, C. S. Gloster, and G. Wilkins, "Longitudinal test of the social cognitive model of choice in engineering students at historically Black universities," Journal of Vocational Behavior, vol. 76, pp. 387-394, 2010.

[20] M. Inda, C. Rodríguez, and J. V. Peña, "Gender differences in applying social cognitive career theory in engineering students," Journal of vocational behavior, vol. 83, pp. 346355, 2013.

[21] R. W. Lent, H.-B. Sheu, D. Singley, J. A. Schmidt, L. C. Schmidt, and C. S. Gloster, "Longitudinal relations of self-efficacy to outcome expectations, interests, and major choice goals in engineering students," Journal of Vocational Behavior, vol. 73, pp. 328$335,2008$. 
[22] G. Hackett, "Role of mathematics self-efficacy in the choice of math-related majors of college women and men: A path analysis," Journal of counseling psychology, vol. 32, p. 47, 1985.

[23] R. W. Lent, M. J. Miller, P. E. Smith, B. A. Watford, R. H. Lim, and K. Hui, "Social cognitive predictors of academic persistence and performance in engineering: Applicability across gender and race/ethnicity," Journal of Vocational Behavior, vol. 94, pp. 79-88, 2016.

[24] R. L. Navarro, L. Y. Flores, H.-S. Lee, and R. Gonzalez, "Testing a longitudinal social cognitive model of intended persistence with engineering students across gender and race/ethnicity," Journal of Vocational Behavior, vol. 85, pp. 146-155, 2014. 